\title{
Age-related differences in the impact of cannabis use on the brain and cognition: a systematic review
}

\author{
Claire Gorey ${ }^{1,2}$ (1) $\cdot$ Lauren Kuhns $^{1,3} \cdot$ Eleni Smaragdi $^{1} \cdot$ Emese Kroon $^{1,3} \cdot$ Janna Cousijn ${ }^{1,3}$
}

Received: 15 August 2018 / Accepted: 3 January 2019 / Published online: 24 January 2019

(c) The Author(s) 2019

\begin{abstract}
The impact of cannabis on the adolescent compared to adult brain is of interest to researchers and society alike. From a theoretical perspective, adolescence represents a period of both risk and resilience to the harms of cannabis use and cannabis use disorders. The aim of this systematic review is to provide a critical examination of the moderating role of age on the relationship between cannabis use and cognition. To this end, we reviewed human and animal studies that formally tested whether age, adolescent or adult, changes the relationship between cannabis exposure and cognitive outcomes. While the results of this review do not offer a conclusive answer on the role of age, the novel review question, along with the inclusion of both human and animal work, has allowed for the formation of new hypotheses to be addressed in future work. First, general executive functioning seems to be more impaired in adolescent frequent cannabis users compared to adult frequent cannabis users. Second, age-effects may be most prominent among very heavy and dependent users. Third, craving and inhibitory control may not decrease as much post-intoxication in adolescents compared to adults. Lastly, adolescents' vulnerability to reduced learning following cannabis use may not persist after sustained abstinence. If these hypotheses prove correct, it could lead to important developments in policy and prevention efforts.
\end{abstract}

Keywords Cannabis $\cdot$ Development $\cdot$ Cognition $\cdot$ Brain $\cdot$ Adolescence $\cdot$ Systematic review

\section{Introduction}

The risks and benefits of cannabis is an active research area due to widespread trends in legalization. Across European and North American countries, higher perceived availability

Lauren Kuhns and Eleni Smaragdi contributed equally to this manuscript and are, therefore, both considered second authors.

Electronic supplementary material The online version of this article (https://doi.org/10.1007/s00406-019-00981-7) contains supplementary material, which is available to authorized users.

Janna Cousijn

j.cousijn@gmail.com

1 Department of Psychology, Addiction Development and Psychopathology (ADAPT) Research Center, University of Amsterdam, P.O. box 15916, 1001 NK Amsterdam, The Netherlands

2 Dynamics of Externalizing (DEXTER) Lab, Department of Psychology, University of South Florida, Tampa, FL, USA

3 The Amsterdam Brain and Cognition Center (ABC), University of Amsterdam, Amsterdam, The Netherlands of cannabis is observed to be a robust predictor of higher frequency of use [1]. This lends concern that legalization could result in higher cannabis use and more harm particularly for adolescents-an often touted 'vulnerable' population [2]. With growing frequencies of use, it is essential to understand the nature and scope of the risks of adolescent cannabis use. Moreover, understanding the potentially differential impact of cannabis on the adolescent compared to the adult brain could provide valuable information for prevention and policy efforts. The central aim of this systematic review is to investigate whether age influences the effect of cannabis on cognition and the brain. To address this, we reviewed studies that formally tested whether age changes the relationship between cannabis exposure and cognitive outcomes.

While public lore is that the adolescent brain is highly vulnerable to drugs, the scientific literature remains mixedwith evidence of heightened risk and of heightened resilience. Adolescence is a period characterized by a hyperactive limbic system, involved in reward, motivation, and affective learning $[3,4]$. Taken together with slower, protracted development of behavioral control [5, 6], adolescents exhibit increased behavioral approach toward rewards and 
less behavioral withdrawal even when met with aversive consequences [4]. This is thought to facilitate the relative fast formation of maladaptive addictive behaviors during adolescence [7]. Moreover, since the adolescent brain is still developing, adolescent cannabis use may be associated with enhanced negative effects on brain structure and function [8], particularly in areas that are strongly tied with the pharmacological effects of cannabis [2]. These factors may render adolescents more vulnerable to the development of persistent substance use disorders (SUDs) and might worsen cognitive outcomes $[9,10]$. Indeed, early-onset, compared to late-onset, cannabis users experience decreased executive functioning [11] and are approximately $2-5$ times more likely to experience cannabis dependence or other types of drug dependence later in life [9].

On the other hand, adolescents may also exhibit heightened resilience to the effects of cannabis. Resilience can be defined as a process of overcoming the risk-related factors through the presence of protective factors. There are several unique characteristics of adolescence that could mitigate the impact of risk-related factors. As with all SUDs, most adolescent-onset cannabis use disorders (CUDs) naturally resolve over time without treatment [12]. Therefore, increased risk for developing CUDs is eventually turned into increased resilience to persistent cannabis use-related problems. High brain plasticity during adolescence might play a central role in this resilience, as evidenced by other types of adolescent-specific resilience (e.g., high rates of recovery after brain trauma) [12]. Furthermore, social factors, such as planning to attend college [13] and parental involvement in school [14], have mitigated the effects of risk-related factors on substance use outcomes in adolescence. In this sense, risk and protective factors during adolescence may function synergistically, thereby building heightened resilience to CUDs and other adverse cannabis-related outcomes in adolescents compared to adults. These competing hypotheses of risk or resilience raise the question of whether cannabis is truly more harmful for the adolescent brain.

Several reviews have addressed this question by exploring the effect of cannabis on adolescents' neural and cognitive functioning [2, 15-22]. Focusing on mainly human work, in the past five years, there have been five qualitative $[2,15,17,18,20]$, two systematic qualitative [16, 22], and two meta-analytic and systematic reviews [19, 21]. Although, the age windows of early versus late onset varied widely $[17,18,22]$, the conclusions were similar. Specifically, earlier onset cannabis use, compared to lateonset, led to increased neurodevelopmental disruptions that resulted in functional and/or structural changes [22]. On the other hand, Blest-Hopley et al. [21] and Scott et al. [19] conducted systematic and meta-analytic reviews that investigated both adolescents and young adult cannabis users, and the results were inconsistent with these past reviews. Blest-Hopley et al. [21] showed that both adolescent and adult cannabis users exhibited deficits compared to controls but in different brain areas, suggesting agerelated risks at both developmental periods [21]. Additionally, Scott et al. [19] found small but significant effect sizes for the relationship between heavy cannabis use and cognition in both adolescents and young adults. Moreover, this effect was not moderated by age group or age of onset [19].

These reviews provide valuable information regarding age-related effects of cannabis on cognition within adolescents and young adults but not between adolescents and adults. Reviews that compared early and late-onset cannabis users often included studies that only compared early and late onset within adolescence, not compared to adulthood. Similarly, Scott et al. [19] and Blest-Hopley et al. [21] included studies that only compared cannabis using adolescents against non-using adolescents or compared cannabis using adults against non-using adults. This addresses the question of whether there is a difference in brain function and cognition for cannabis users, compared to non-users, in adolescents and in adults separately (and whether the effect sizes differed). As such, a systematic review of studies that directly compared age groups is missing and the question of whether adolescents compared to adults exhibit enhanced risk or resilience to the neurocognitive effects of cannabis use remains unanswered.

In the current review, we aimed to extend previous work by examining two critical questions: does the relationship between cannabis use and cognition differ between adolescents and adults (i.e., interaction of cannabis exposure by age) and if so, do the effects vary based on cannabis use history and intoxication state? To address these questions, we systematically reviewed rodent and human studies that directly compared age groups and treated age as a moderator for the relationship between cannabis exposure and cognition. By including rodent studies, several confounds in reviews that only included human studies can be addressed. First, human work is complicated by the fact that cannabis exposure is inherently confounded with age. For example, early-onset users, compared to lateonset, may show a greater vulnerability simply because they used cannabis for a longer period. Second, human studies rarely incorporate a prolonged abstinence period and for ethical reasons, this cannot be randomly assigned. By having control over cannabis exposure, abstinence periods, and confounding variables, animal studies allow for more causal inferences into neurobiological processes. By incorporating both rodent and human studies, we are uniquely positioned to answer the question of whether there is greater risk for or resilience to cognitive decrements in adolescent cannabis users compared to adults-a novel review approach and question. 


\section{Method}

\section{Study inclusion criteria and search strategy}

We followed the Preferred Reporting Items for Systematic Reviews and Meta-Analyses (PRISMA) guidelines for the current systematic review [23]. A MedLine, Cochrane Library, and Psyc Info search was conducted during July of 2018 with terms related to cannabis, cognition, adolescence/ adulthood, and study type (see Appendix S1 for full search strategy and syntax). One author (CG) examined whether retrieved studies met or did not meet our inclusion criteria and another author (ES) conducted a random check of $1 / 3$ of the articles. If there was a discrepancy after initial coding, four authors (CG, ES, LK, and JC) reviewed the article to reach a consensus.

Inclusion criteria were: (1) human samples must have included both adolescents younger than 18 and adults older than 18, and rodent samples must have included adolescent (post-natal day 25-42 for rodents) and adult (greater than postnatal day 43 for rodents) rodents; (2) must have explored cannabis exposure as the independent variable and cognitive outcomes as the dependent variable; (3) the analyses must have included an age by cannabis exposure interaction on cognition, with age being explored either categorically (adolescent or adult) or continuously; (4) must have administered measures during adolescence or adulthood, not retrospectively; (5) must have used primary quantitative data collection methods (e.g., no case-studies, review papers); (6) must have solely looked at cannabis-related factors as the independent variables (e.g., did not explore cannabis-related factors in individuals with psychosis); (7) must be written in English; (8) must be published in a peer-reviewed journal before July 19th, 2018 (see Fig. 1 for a detailed screening process). Of note, we excluded studies that assessed cannabis exposure retrospectively (i.e., inclusion/exclusion criteria \# 4). The studies excluded for this reason were predominantly early-onset cannabis use studies. We excluded these because age-onset variables are often inaccurate [24], with recalled age of onset increasing as an individual's historical age increases [25]. Additionally, we decided to exclude unpublished work (i.e., inclusion/exclusion criterion \# 8) to ensure that the quality of the work included in our review passed the standards of independent peer review.
Records identified through database searching $(n=2,124)$
Additional records identified through Google Scholar

$$
(n=1)
$$

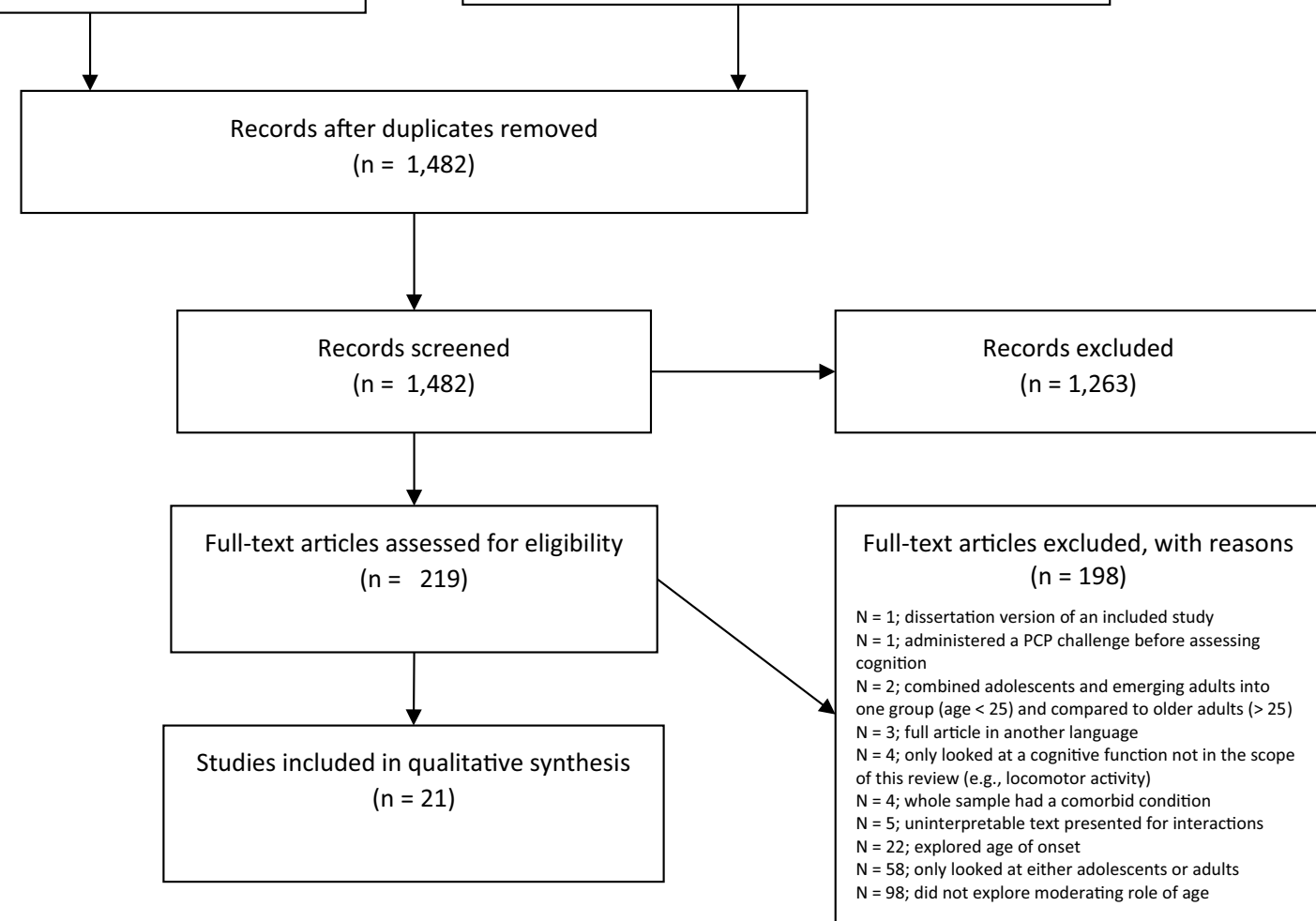

Fig. 1 PRISMA flow diagram detailing our screening process 
In humans, we defined cognition as any construct that typically falls within the bounds of standard neuropsychological testing (e.g., processing speed, executive functioning). We also included more distal constructs of cognition, like craving and impulsivity, because they play a prominent role in learning, drug use, and addiction (for reviews see [26, 27]). In rodents, we defined cognition as attention, learning, and memory (see Fig. 2 for processes considered), in line with a seminal review paper [28]. However, we did not discuss findings related to the behavioral phenotype of the animal, such as locomotion and anxiety. Within the included studies, peripheral findings that did not relate to cognition were excluded from review. Additionally, reported effects irrelevant to the exploration of age as a moderator for cannabis and cognition were not included. Although, we did report post hoc testing that helped to determine the direction of the interaction.

\section{Results}

\section{Study search}

Our search resulted in 1482 studies once duplicates were excluded (see Fig. 1). 1263 studies were excluded after reviewing the abstracts. 198 studies were excluded after

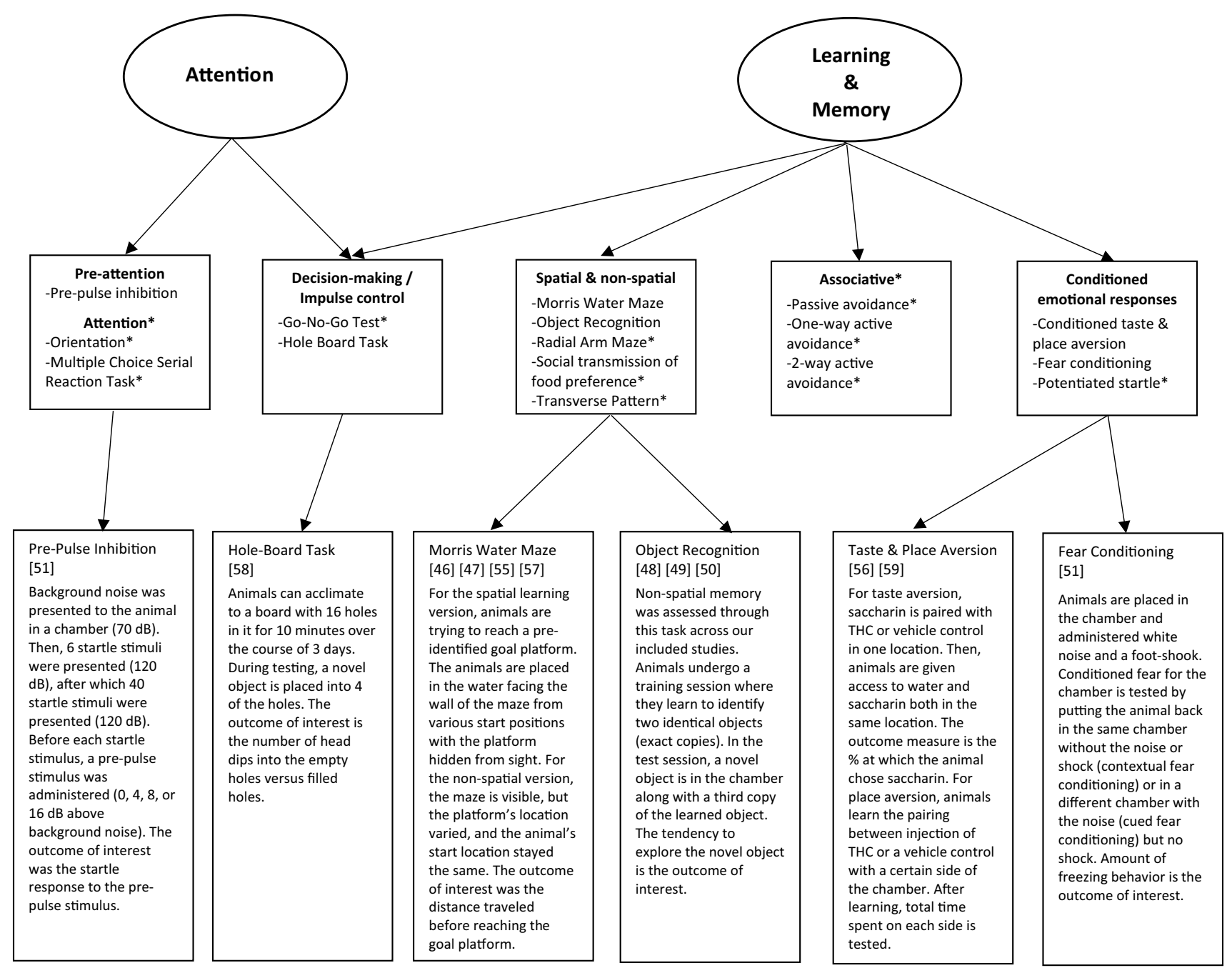

Fig. 2 Cognitive domains assessed in rat studies across the literature are in circles, as described in a seminal review paper (see [28]). The second layer of boxes contains the narrower cognitive function along with the behavioral tasks typically used to assess that function. Asterisks represent cognitive functions or tasks that were not assessed or used in the included studies covered in this review. The third layer of boxes contains short descriptions for the tasks used across the included studies. The numbers in brackets are the citation for the study/studies that used the task. It should be noted that these are general overviews of the tasks used across our included studies; therefore, there may be slight variations in how the task was administered. Additionally, even though Pre-Pulse Inhibition is mostly used in schizophrenia research to assess sensorimotor deficits, here we mostly used and interpreted this measure as a reflection of pre-attention processes 
reviewing the full text. After this process, 21 rodent $(N=15)$ and human $(N=6)$ studies were included in our final qualitative review. The characteristics of the final studies are detailed in Tables 1 and 2. In addition to all standard reporting practices according to PRISMA [23], we also listed the qualitative description of the effect sizes in the studies and evaluated the quality of evidence for human work based on our own objective criteria. We included this information in Table 1.

All authors independently reviewed and rated the quality of human evidence: (1) strong level of causality: longitudinal studies with a comparison of adolescent and adult values and that included relevant covariates; (2) moderate level of causality: studies that were longitudinal with a comparison of adolescent and adult values without accounting for relevant covariates or cross-sectional human studies with matched adult and adolescent groups that considered relevant covariates; (3) weak level of causality: studies that were cross-sectional but did not have matched adolescent and adult groups and/or did not consider relevant covariates. Notably, we did not rate the quality of rodent work. Given the enhanced experimental control, the quality was very similar (and strong) across rodent studies. In the discussion, we did provide an overview of the limitations that reduce the generalizability of the rodent work overall.

\section{Human studies on cannabis and cognition}

Human studies explored the role of age on the relationship between some form of cannabis use history (e.g., dependence/amount past use) and cognition or alternatively, on the relationship between cannabis intoxication and cognition. These two types of studies are discussed separately below.

\section{Age, history of repeated cannabis exposure, and cognition}

Four human studies assessing the relationship between history of repeated cannabis exposure and cognition met our inclusion criteria (see Table 1 for study characteristics). Meier et al. [29] investigated participants over the course of 33 years and were interested in the persistence of cannabis dependence (total number of times a cannabis dependence diagnosis was met across 5 study waves; 1,2 , or $3+$ ) by age of first diagnosis (before age 18 and after age 18) on change in intelligence quotient (IQ). Past year cannabis dependence was assessed through a diagnostic interview at age 18, 21, 26, 32 and 38, and IQ was assessed before and after the initiation of cannabis use at age 7, 9, 11, 13 and 38 (Wechsler Intelligence Scale for ChildrenRevised or for Adults-IV). There were no effects of age of first diagnosis on change in IQ (i.e., post-cannabis IQ minus average IQ before cannabis initiation) with only 1 or 2 diagnoses of cannabis dependence. However, individuals that had a dependence diagnosis at 3 or more waves and that met their first diagnosis before age 18 experienced a 0.55 standard deviation reduction in IQ compared to those that had a first diagnosis after age 18. Meier et al. [29] repeated this analysis using weekly cannabis use (instead of diagnosis of dependence) before or after age 18 on change in IQ and results were similar [29].

Like Meier et al. [29], three separate studies found agerelated cognitive vulnerabilities in adolescents but only for heavier cannabis users. More specifically, Scott et al. [30] administered the Penn Computerized Neurocognitive Battery to almost daily and daily cannabis users $(\sim 3-7$ times per week) and discovered that adolescents (i.e., ages 14-17) compared to adults (i.e., ages 18-21) exhibited lower executive control, consisting of sustained attention and working memory subtests, in comparison to the non-user control group. However, this age-related deficit did not extend to weekly cannabis users (1-2 times per week or less) when compared to the non-user group. Additionally, there was no age by cannabis effect on other cognitive domains, including memory (i.e., verbal episodic, face, and spatial episodic memory), complex cognition (i.e., mental flexibility, language reasoning, nonverbal reasoning, and visuospatial ability), or social cognition (i.e., emotion identification, emotion differentiation, and age differentiation). However, weekly cannabis users, but not almost daily or daily users, performed better than non-users in executive control, memory, and social cognition, suggesting a potential positive effect for weekly cannabis users regardless of age [30].

Lee et al. [31] discovered that adolescents, compared to adults, in outpatient therapy for CUDs showed a smaller reduction in bias toward immediate, smaller cannabis and monetary rewards from pre to post treatment, as measured by a delay discounting task. Adolescents and adults both showed decreased reductions in bias towards cannabis compared to money from pre to post treatment, suggesting no age-related differences in delay discounting based on the type of reward presented. These results suggest that adolescents with CUDs, compared to adults, exhibit less positive change in impulsive responding to cannabis and money from pre to post-treatment [31]. Lastly, although not one of their central questions, Albertella et al. [32] cross-sectional study analyzed the effect of continuous age (15-24 years old) by cannabis use frequency, defined as less than once a week or more than once a week over the past 6 months, on the ability to detect relevant targets amongst distracting stimuli in a location-based negative priming task. There was no main effect of age on negative priming. However, there was an interaction between age and cannabis use frequency such that weekly younger users showed lower negative priming scores, or lower accuracy in detecting relevant targets amongst distracting stimuli, compared to weekly older users. This effect did not extend to monthly users, suggesting 


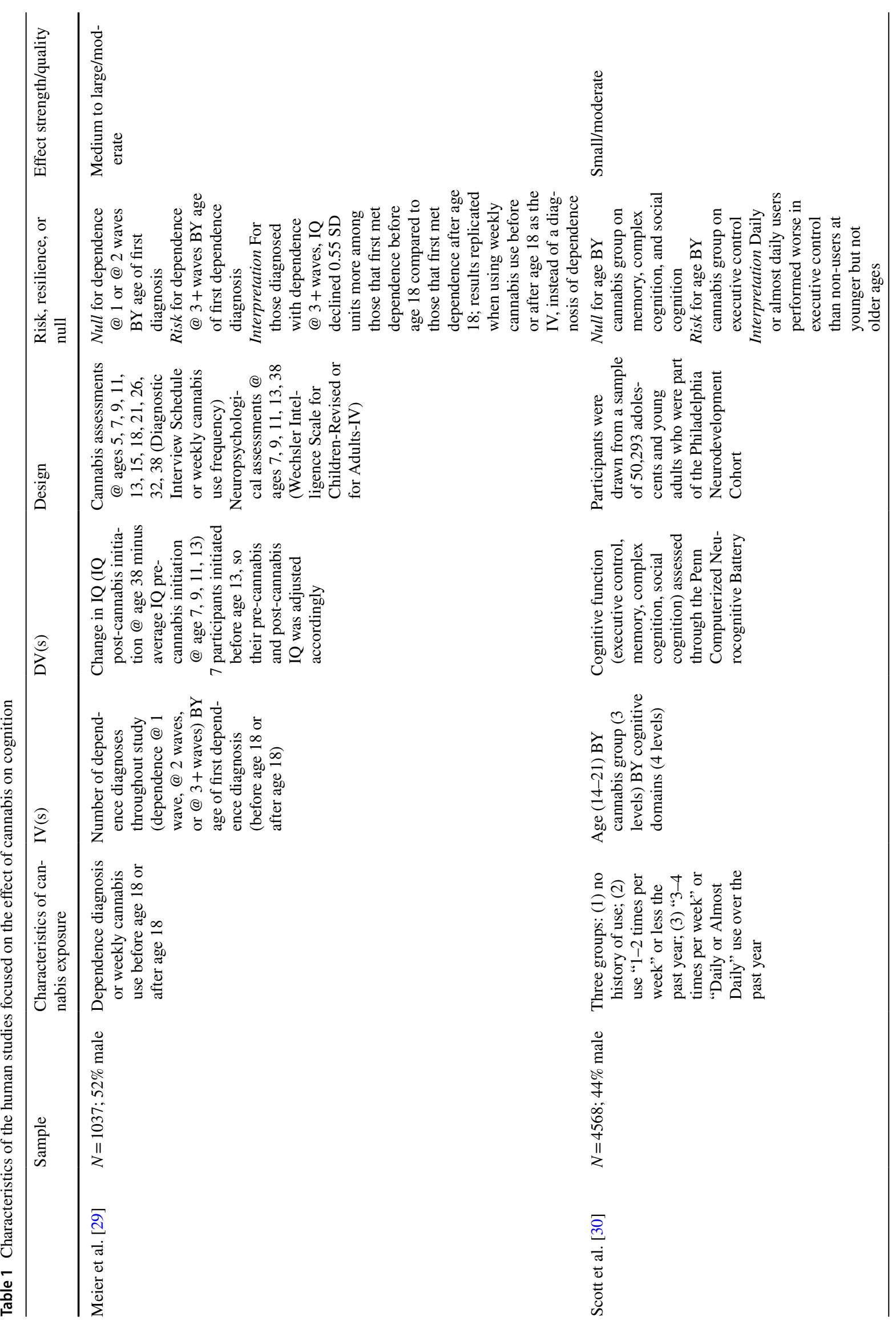




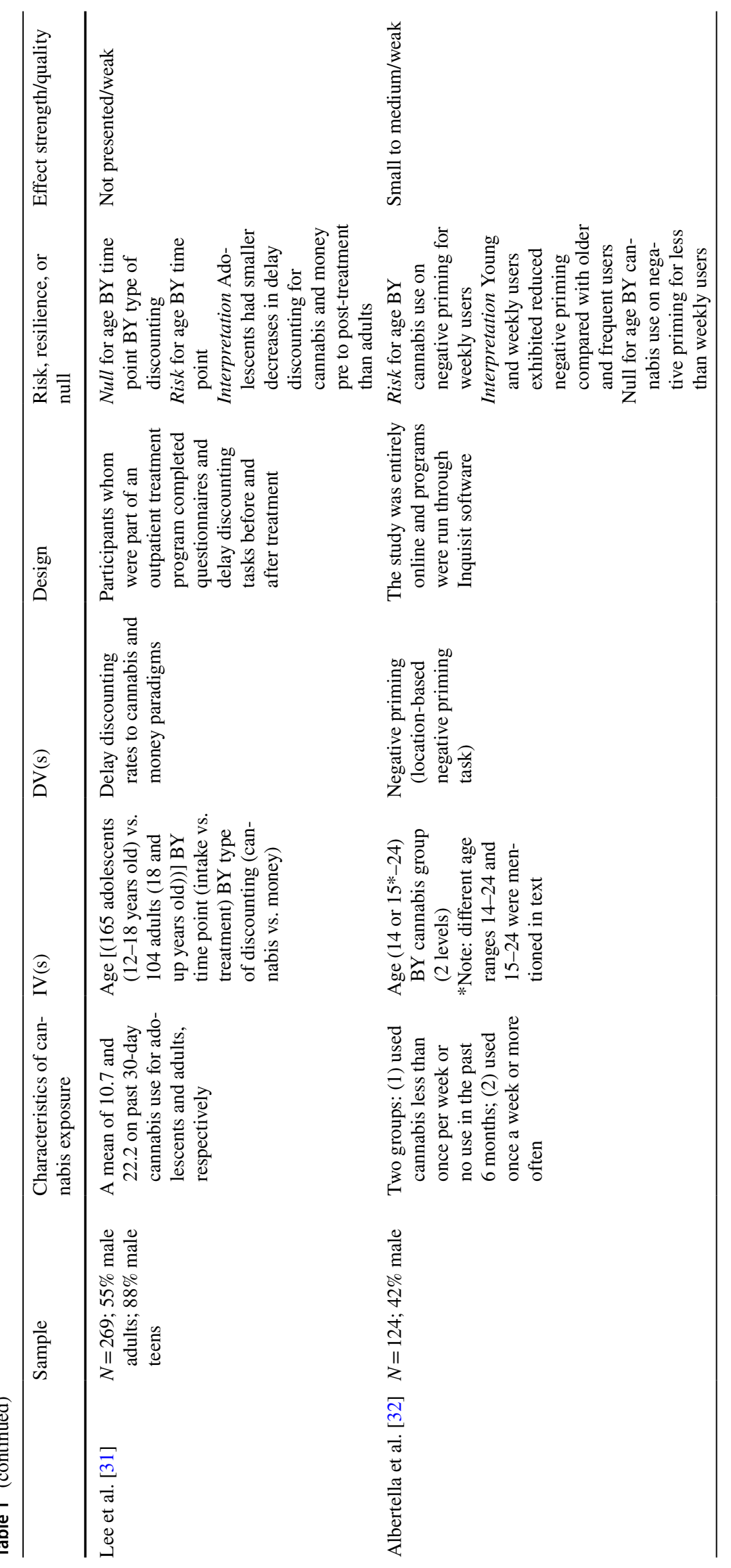




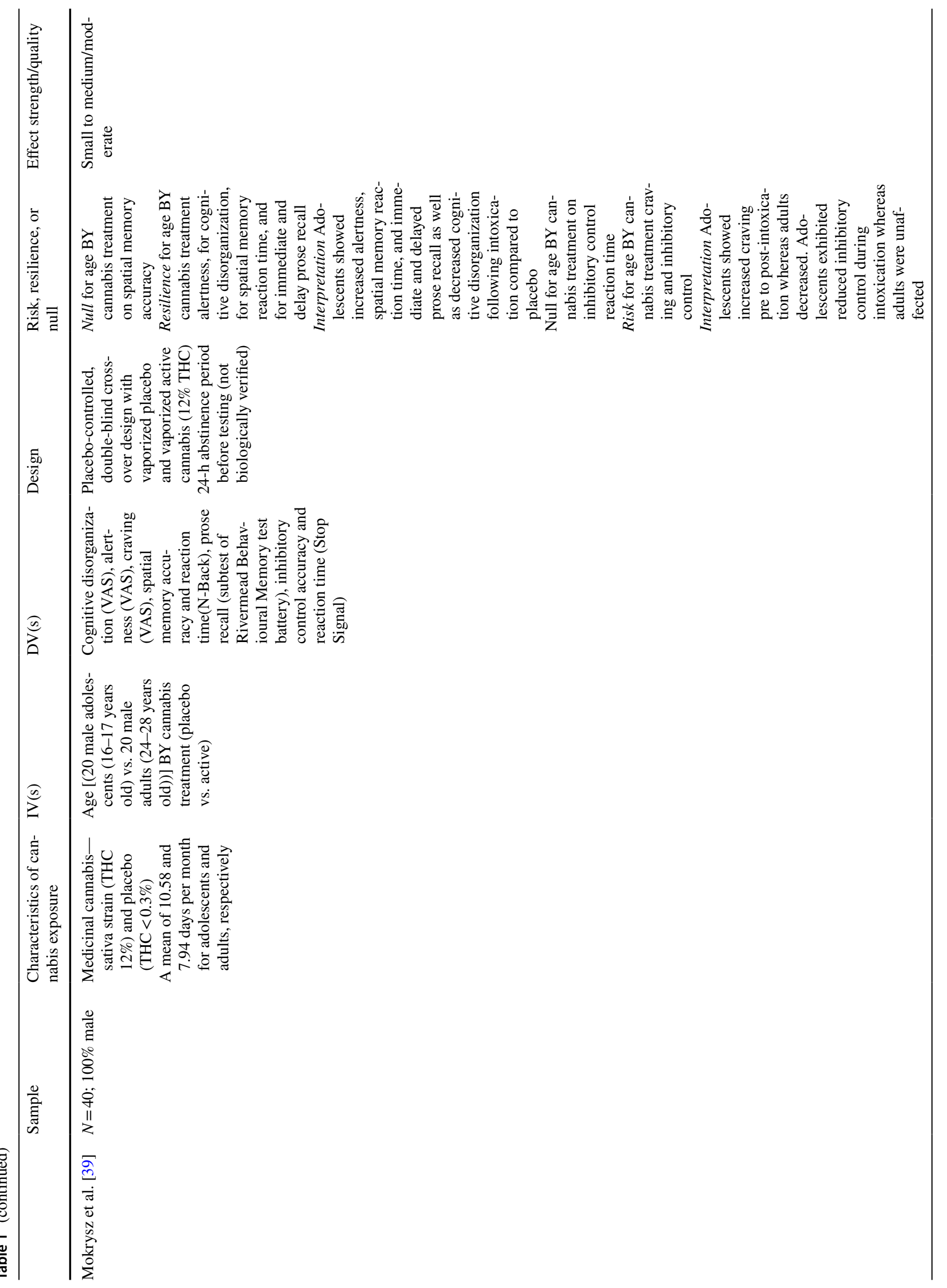




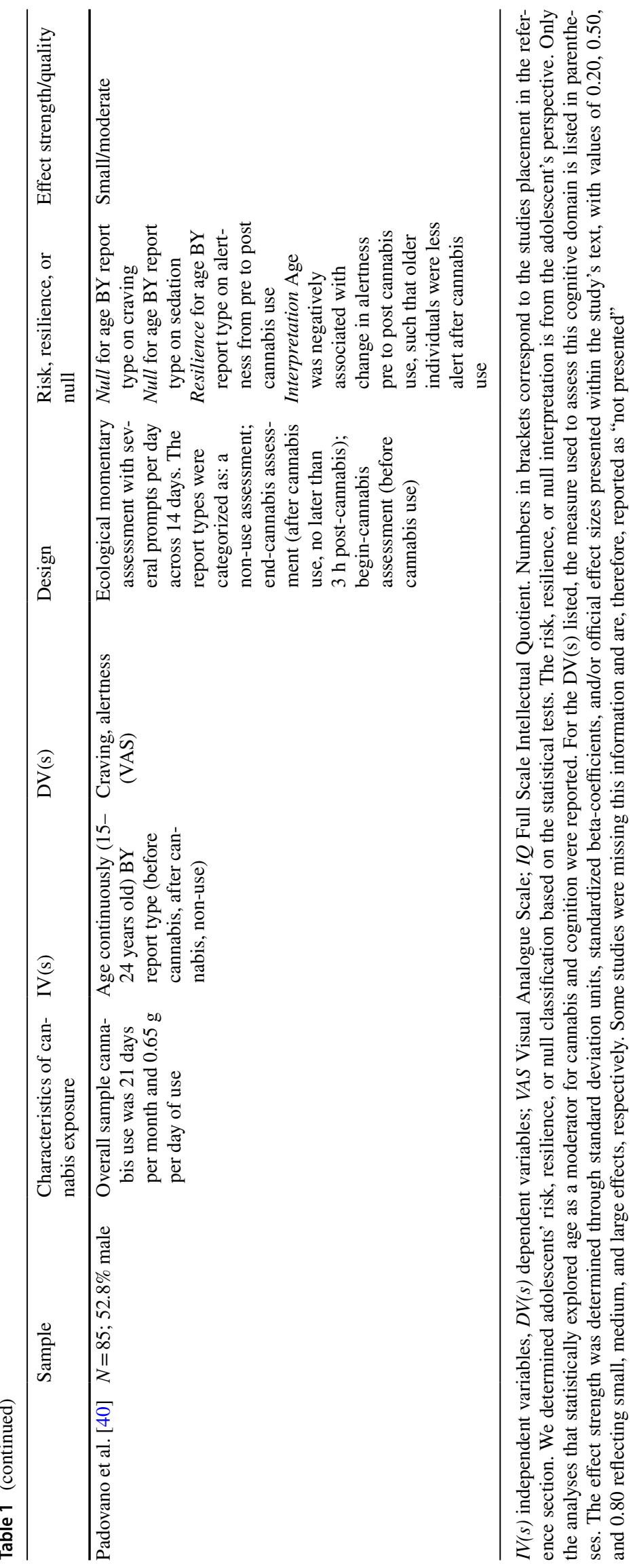




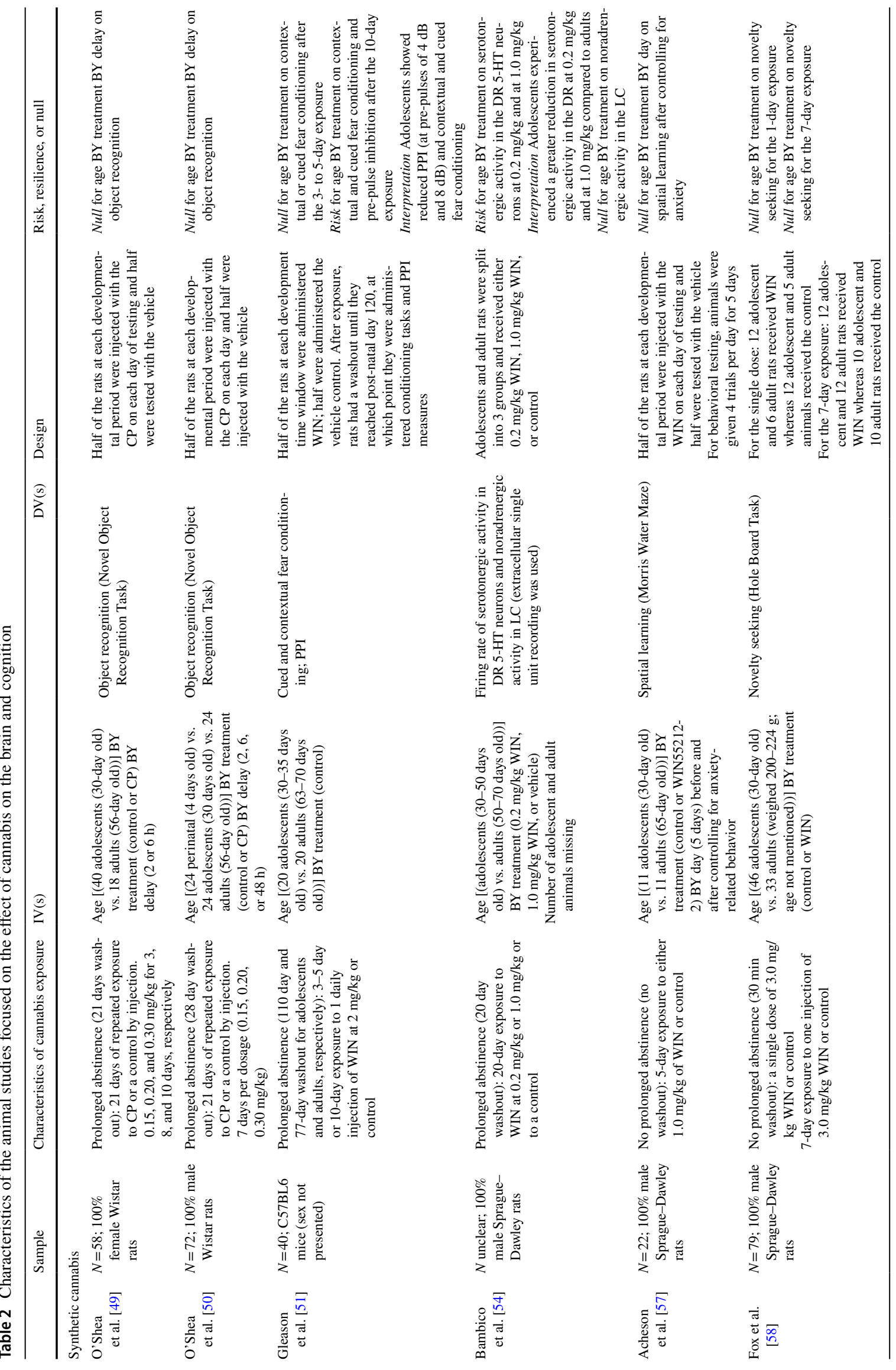




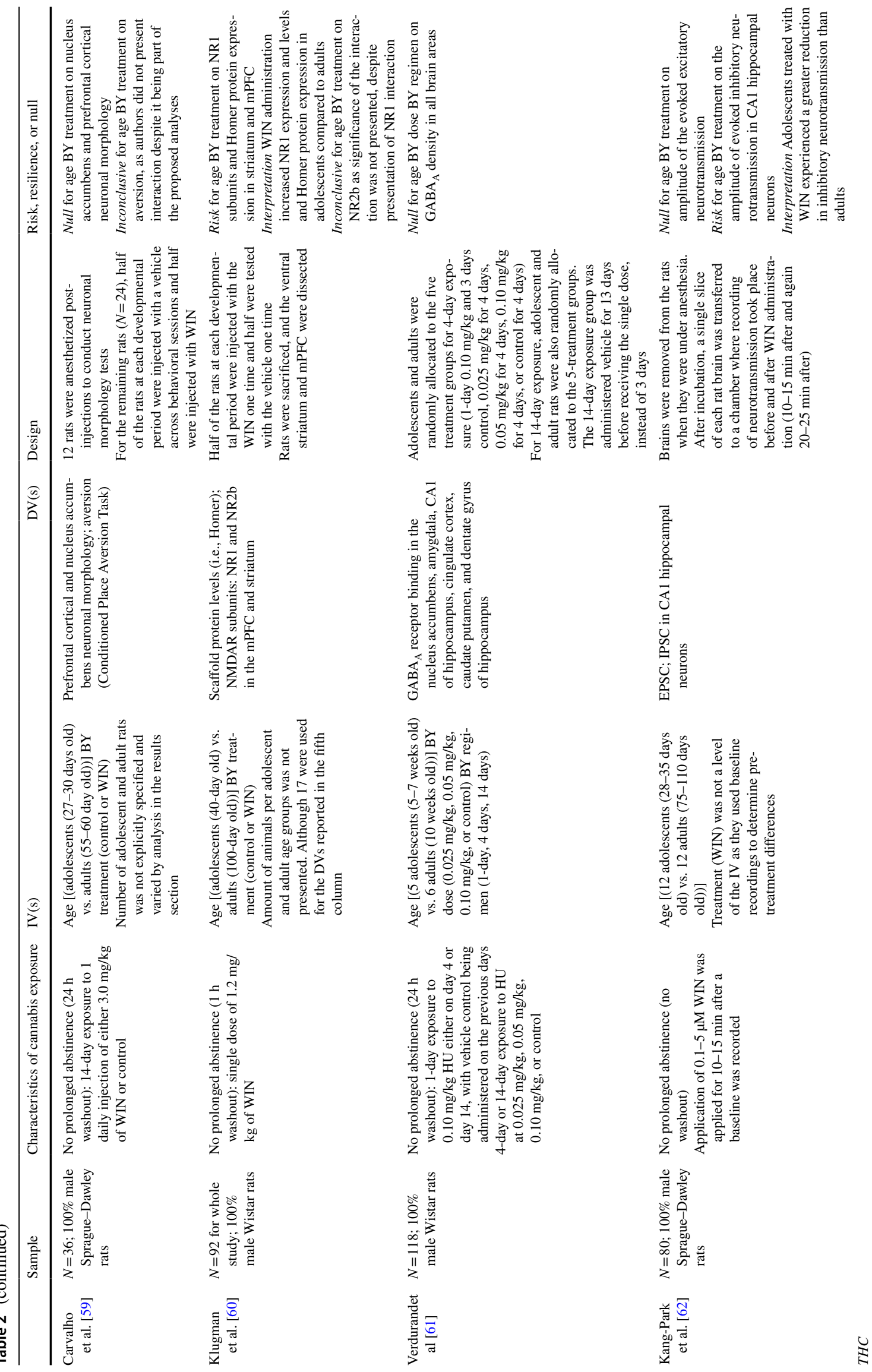




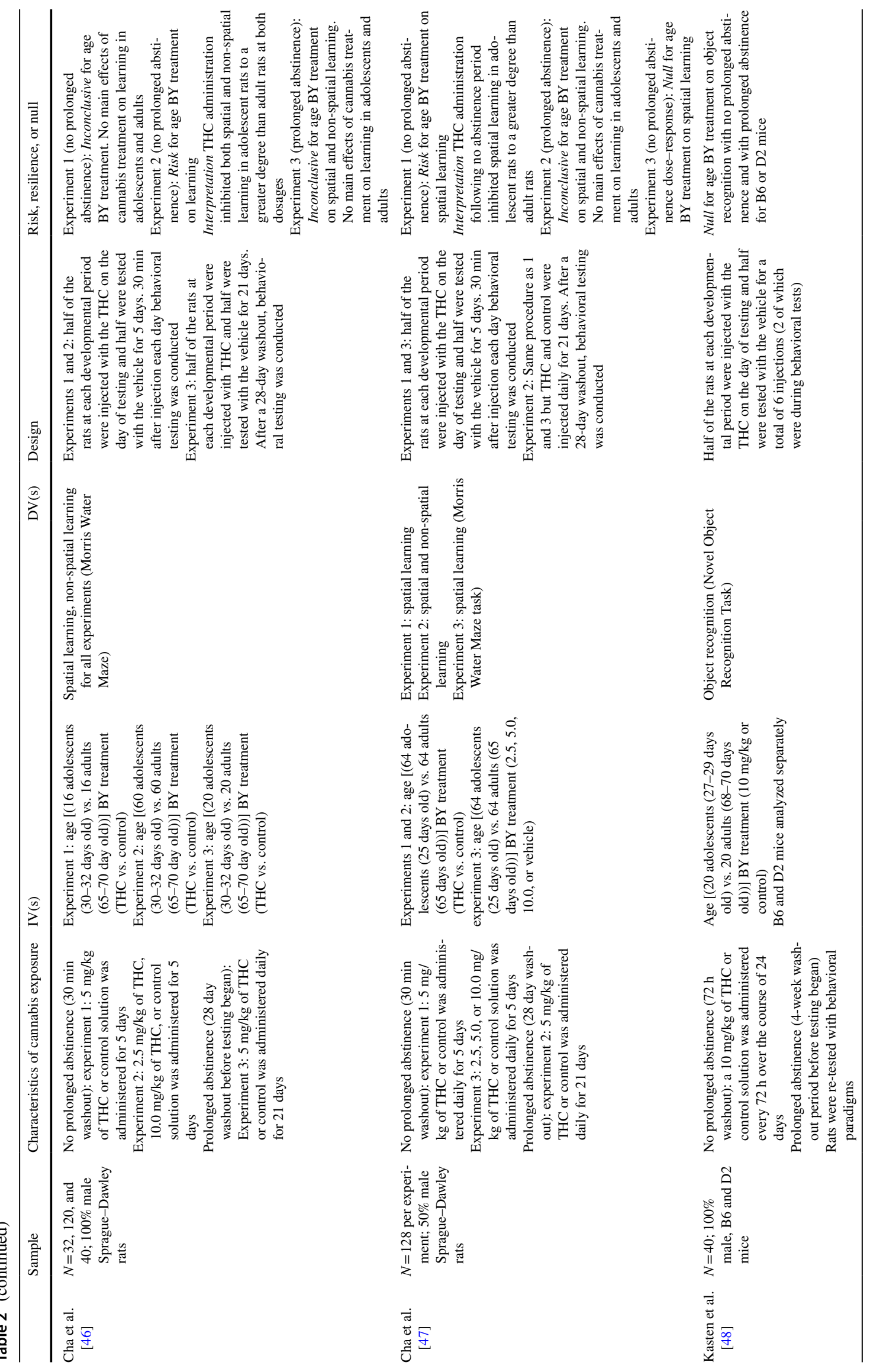




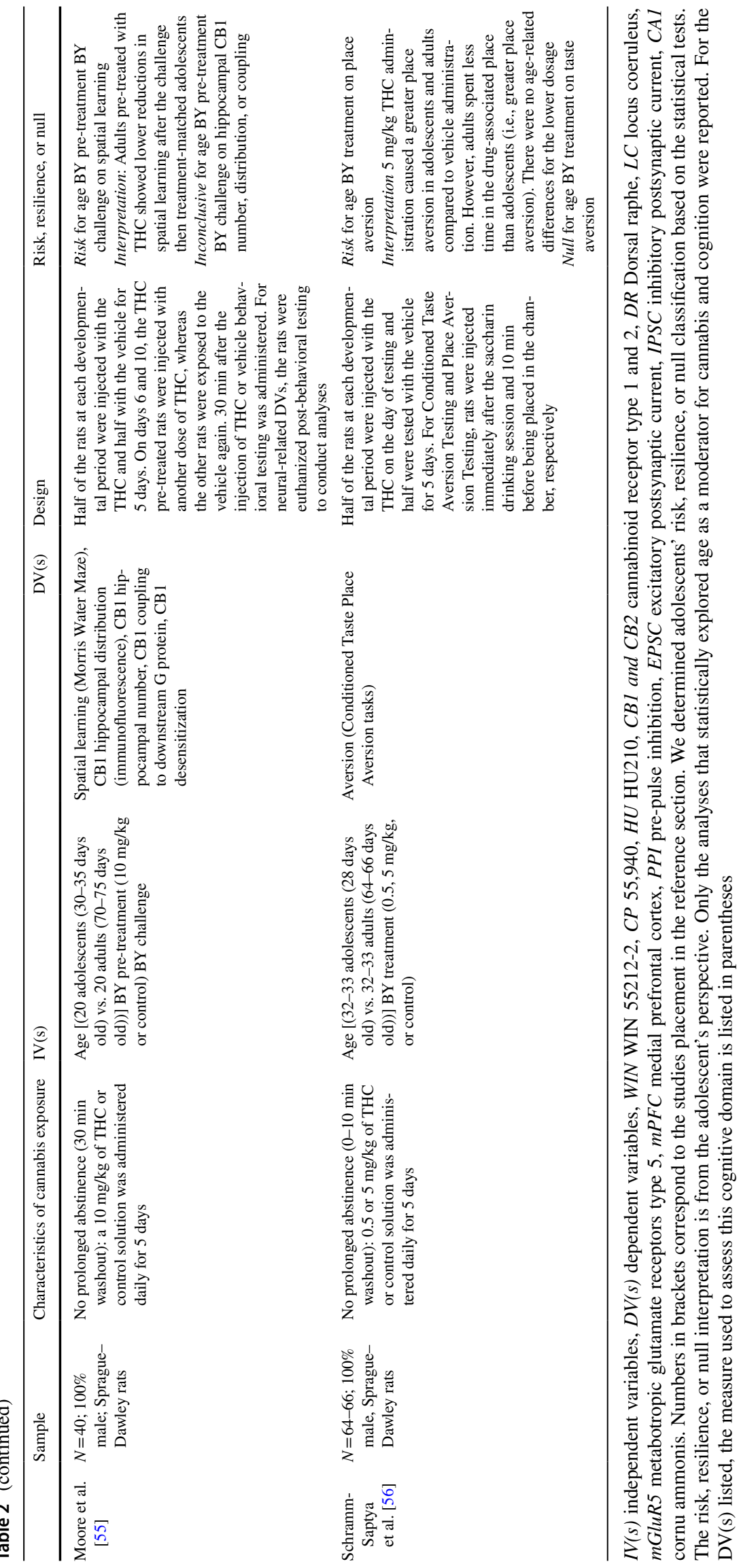


compromised inhibitory control in younger, weekly users specifically [32].

We categorized these four studies as only meeting a weakto-moderate level of causality. Additionally, we assessed the strength of the significant age by cannabis interactions with the reported standardized beta coefficients or z-scores. The effects were small ( 0.20 or less) for Scott et al. [30], small to medium (between 0.20 and 0.50 ) for Albertella et al. [32], and medium to large (between 0.50 and 0.80 ) for Meier et al. [29].

Meier et al. [29] study was classified as a moderate level of causality. The analyses were between-subject, and thus, it could be that other variables not accounted for are associated with adolescent-onset cannabis dependence and the timevarying effect of IQ [29]. For instance, two commentaries concluded that the effect could be accounted for by a simulation model of the confound, socioeconomic status [33], and by personality traits [34]. These arguments were later rebutted by a commentary from the original authors, who showed that effects replicated when only looking at subjects in the middle socioeconomic class and after accounting for self-control [35]. However, since the age-related analyses are based on a between subject factor, the study was not classified as having a strong level of causality. Scott et al. [30] study was classified as a moderate level of causality as well. Their effects were robust beyond numerous confounds, including socioeconomic status. However, given the crosssectional nature, it is impossible to determine if the observed deficits were pre-existing [30].

Lee et al. [31] study was classified as weak level of causality. Adolescents showed a smaller decrease in bias toward immediate cannabis-related and monetary rewards from pre to post treatment. These results could indicate poorer cognitive recovery in adolescents. If true, it is surprising given that adults used twice the amount of cannabis than adolescents in their sample (22.2 days for adults vs. 10.7 days for adolescents; [31]). Alternatively, though, it may mean that adolescents are resistant and unmotivated to therapy in general. Adolescents are rarely self-referred to treatment for CUDs. Moreover, it has been shown that motivation, pretreatment expectations of positive change, and therapist-client relationship account for most of the variance in client outcomes, with actual treatment modality only accounting for $1 \%$ of the variance in symptom reduction [36, 37]. Additionally, it could also be that adolescents may have reached a developmental ceiling for delay discounting with treatment, and therefore, could not reach the same level of reduction in delay discounting as adults. This is in line with literature suggesting that adolescent controls, compared to adult controls, displayed higher rates of delay discounting to money [38]. Without control groups for adolescents and adults within their investigation, it is impossible to determine how age-matched peers without CUDs would perform in delay discounting tasks. Along with these issues of interpretation, we also classified the quality of the evidence for causality as weak due to several methodological issues. Specifically, the unmatched groups regarding past cannabis use (22.2 days of cannabis use per month for adults, 10.7 for adolescents), motivation or expectancy for therapy, gender ( $88 \%$ male for adolescent, $55 \%$ male for adults), and group size ( $N=165$ for adolescents, $N=104$ for adults), as well as the use of different treatment modalities across groups all limited the study's evidence of causality. Similarly, Albertella et al. [32] study was classified as weak evidence for causality because (sub) acute effects of cannabis intoxication might have confounded the findings as $37 \%$ of the weekly and $3 \%$ of the less than weekly users used cannabis within the past $24 \mathrm{~h}$.

Overall, these four studies suggest that adolescent weekly to daily cannabis users experience greater reductions in general executive functions like working-memory and attention, which may in turn affect cognitive tasks that rely on these functions. The effect in one study seems to extend to IQ in individuals with persistent cannabis dependence (diagnosis at 3 or more study waves) with a first diagnosis before age 18. This further supports the hypothesis that age-related deficits in executive functioning and Full-Scale IQ are probably most noticeable in the most heavy and problematic cannabis users. However, it is unclear if these age-related deficits would extend beyond a prolonged abstinence period. Specifically, none of these studies had a standardized abstinence period. Therefore, intoxication levels during the cognitive assessment might vary between participants within studies as well as between studies. For the studies of Scott et al. [30] and Lee et al. [31], it is unclear whether (sub)acute effects of intoxication could have affected the results, as no measure of recent cannabis use was included. Meier et al. [29] and Albertella et al. [32] did assess past 24-h cannabis use and took this into account in part of their analyses. Meier et al. [29] showed that their main results did not change significantly after exclusion of past 24-h cannabis users. Albertella et al. [32] showed that across all participants, past 24-h cannabis use was not a significant predictor of their main outcomes measure, negative priming, but did not take into account the existing group difference in past $24 \mathrm{~h}$ use. Given this confound, the limited amount of studies, and the strength and quality of evidence, results should be considered preliminary.

\section{Age, cannabis intoxication, and cognition}

Two human studies, that met our inclusion criteria (see Table 1 for study characteristics), conducted studies on the effects of cannabis intoxication in current adolescent and adult cannabis users. Notably, only one of these studies [39] had a standardized abstinence period, although it was only $24 \mathrm{~h}$ and was not biologically verified. Mokrysz 
et al. [39] administered both vaporized cannabis (12\% THC) and a placebo control, in separate sessions, to a matched sample of 20 male adolescents (16-17 years old; mean of 10.58 days of cannabis use per month) versus 20 male adults (24-28 years old; mean of 7.94 days of cannabis use per month). Interestingly, adolescents showed signs of both risk and resilience. Adolescents showed less memory impairments, both immediate and delayed. That is, adults showed twice as large of a reduction in delayed prose recall following intoxication, compared to placebo, as well as lower immediate prose recall than adolescents. Moreover, adolescents did not show a difference in reaction time to a spatial N-back working memory task during intoxication compared to placebo; whereas, adults were significantly slower during cannabis intoxication compared to placebo. There were no age-related differences for spatial N-Back accuracy. Lastly, adults reported significantly higher cognitive disorganization and significantly lower alertness on a visual analogue scale while intoxicated than adolescents.

In contrast to the working memory findings, adolescents showed heightened risk for craving (measured by a visual analogue scale) and accuracy, but not reaction time, in an inhibitory control task (measured by a stop signal task). Specifically, pre- to post-intoxication craving increased in adolescents but decreased in adults. Moreover, adults' inhibitory control score was unaffected by cannabis; whereas, adolescents showed reduced inhibitory control accuracy when intoxicated [39]. In a partial replication of Mokrysz et al. [39], Padovano et al. [40] conducted an Ecological Momentary Assessment in cannabis users, aged 15-24 years old, who averaged roughly 21 days of cannabis use per month and $0.65 \mathrm{~g}$ per use day. The researchers tracked participants across 14 days and administered craving and alertness measures via a wireless device that delivered several prompts each day. Researchers then categorized their assessments as during non-use days, before cannabis use, or after cannabis use [40]. In line with Mokrysz et al. [39] age was negatively associated with change in alertness post-cannabis use, such that younger individuals were more alert relative to older individuals. This effect did not extend to sedation. Unlike Mokrysz et al. [39], there was no effect of age on craving from pre- to post-cannabis use.

We rated both studies as moderate evidence of causality, given the sound experimental design and control of confounding variables. The strength of the effects for Padovano et al. [40] were small (less than 0.20), as determined by the presented standardized beta coefficients. For Mokrysz et al. [39], the strength of the effects were medium $(0.20-0.50)$ for alertness, immediate/delay prose recall, reaction time to the N-back task, and inhibitory control accuracy and were small (less than 0.20) for craving, as determined by the presented eta-squared values. Given Mokrysz et al. [39] finding of reduced cognitive impairment post-intoxication in adolescents, one could hypothesize that this would be beneficial to negative outcomes of acute intoxication (e.g., risky driving) as well as long-term cognition. Therefore, this resilience effect of cannabis on adolescents' cognition when intoxicated contradicts the risk effects observed in non-intoxicated cannabis users. However, Mokrysz et al. [39] finding for inhibitory control is consistent with the studies reviewed above. This suggests that age effects may vary based on intoxication state and discrepancies may only appear for certain cognitive functions.

In addition, the observed effects of age on craving conflict; however, this could be due to methodological differences across the two studies. Specifically, Mokrysz et al. [39] treated age dichotomously (adolescents vs. adults), whereas Padovano et al. [40] treated age continuously. Furthermore, the cannabis users in Padovano et al. [40] sample exhibited two times higher frequency of cannabis use than Mokrysz et al. [39] sample. These methodological influences may have had more of an impact in their discrepant findings particularly because the effect for craving was small in Mokrysz et al. [39] study.

Overall, the results of these two studies of current cannabis users under acute cannabis intoxication appear to contradict each other for craving but not alertness. Additionally, except for the inhibitory control outcome, Mokrysz et al. [39] findings on cognition differed from included human studies that did not incorporate acute intoxication. It is possible that age intersects with acute intoxication differently, thereby leading to distinct patterns with cognitive outcomes compared to non-acute intoxication investigations.

\section{Animal studies on cannabis and cognition}

Across animal work, researchers typically expose rodents to daily cannabinoid injections, including tetrahydrocannabinol (THC), the main compound in natural cannabis, or synthetic cannabis. The reviewed THC administration studies are most relevant to the human studies described above, of which none considered the use of synthetic cannabinoids. Findings for synthetic cannabinoid administration studies do not necessarily directly translate to the effects of natural and synthetic cannabinoids in humans. However, such studies are important because they provide insight into synthetic cannabinoids, which are studied very minimally in human work. Animal studies that tested cognition in repeatedly exposed rodents during THC intoxication are most comparable to human studies that tested cognition post-cannabis intoxication (see $[39,40])$. Rodent studies that tested cognition in repeatedly exposed rodents after prolonged abstinence are most comparable to human studies that tested abstinent cannabis users [31]. In terms of the translation of rodents to human development, rats and mice have similar developmental periods [41] and are considered 10-18-years-old during 
post-natal day 25-42 (i.e., adolescence) and 18-25-year-old during post-natal day 43-65 (i.e., emerging adulthood; [42]). Given the fast development, it is common for researchers to only administer THC or synthetic cannabis for 5 days, which is roughly comparable to 6 months of daily exposure in humans [43].

Researchers studying rodents use a variety of measures designed to match human cognitive functions. In Fig. 2, we present a more in-depth description of the tasks used across the included rodent studies and what domain of cognition they target. Fifteen rodent studies met our inclusion criteria (see Table 2 for study characteristics), of which five administered THC and ten administered a synthetic cannabinoid. Across studies, half of the rodents at each development period were administered the active cannabinoid and half were administered a vehicle control. Synthetic cannabinoid studies used compounds such as CP 55,940 (CP), WIN 55212-2 (WIN), and HU210 (HU). All these compounds interact with both Cannabinoid (CB) 1 and CB2 receptors, like the main active ingredient in natural cannabis (i.e., THC), but are significantly more potent [44, 45]. All included animal studies used rodents and neurocognitive assessments were performed during acute intoxication or after prolonged abstinence. The results of these studies are discussed separately below.

\section{Age, repeated cannabinoid exposure, and cognition after prolonged abstinence}

Three experiments explored the effect of repeated THC exposure on learning and/or memory after prolonged abstinence. To investigate the effects on spatial and non-spatial learning through the Morris Water Maze, Cha et al. [46] administered $2.5 \mathrm{mg} / \mathrm{kg}$ THC or $10.0 \mathrm{mg} / \mathrm{kg}$ THC to male Sprague Dawley rats for 21 days (1 injection per day) [46] and then, extended their design to a mixed-gender sample [47]. For both of their experiments, the authors did not provide statistics for their age by cannabis treatment interaction, despite proposing it in their statistical analyses. However, there were no main effects of cannabis treatment (THC vs. control) on spatial and non-spatial learning in adolescents or adults across both studies [46, 47]. Kasten et al. [48] administered $10 \mathrm{mg} / \mathrm{kg}$ THC or control vehicle to male B6 and D2 mice across 24 days ( 1 injection every $72 \mathrm{~h}$ ) and found no evidence of age-related differences on object recognition, as measured by the Novel Object Recognition test, following repeated THC administration and a 4-week washout period. No overall main effect of treatment emerged across the age groups [48].

Four experiments investigated age-related differences of the effect of synthetic cannabinoid exposure after prolonged abstinence. To investigate working memory, O'Shea et al. [49] administered different dosages of $\mathrm{CP}$ to female Wistar rats for
21 days (1 injection per day). After 21 days of abstinence, there was no age by cannabis treatment effect or main effect of treatment on working memory across age groups, as measured by the Novel Object Recognition Task [49]. In a follow-up study, O'Shea et al. [50] repeated the same protocol in male Wistar rats with a 28-day abstinence period. CP reduced working memory in both adolescents and adult CP-treated rats compared to vehicle, but like their study in females, age did not moderate the effect of CP on working memory [50].

Gleason et al. [51] investigated the effect of WIN administration (3- to 5-day exposure and 10-day exposure) on cued and contextual fear conditioning and sensorimotor gating in adult and adolescent C57BL6 mice. After prolonged abstinence, there was an age by cannabis treatment effect in the 10-day exposure group only. Specifically, adolescent mice showed increased reductions in sensorimotor gating (a measure of attentional abnormalities) and cued and contextual fear-conditioning, while no impairments were found in adult mice [51]. Lastly, Bambico et al. [54] administered WIN to male Sprague-Dawley for 20 days to investigate its effects on the firing rates of serotonergic and noradrenergic activity in dorsal raphe neurons and locus coeruleus neurons (areas involved in appetitive and aversive information processing; see $[52,53])$. After a 20-day washout, they observed a moderating effect of age on serotonergic activity in the dorsal raphe but not on noradrenergic activity in the locus coeruleus. Specifically, adolescents experienced a significantly greater reduction in serotonergic activity in the dorsal raphe compared to adults. While there was no significant interaction for noradrenergic activity, main effects suggested that adolescents, not adults, experienced significant enhancement in noradrenergic firing rates in a dose-dependent manner following cannabis exposure [54].

Following prolonged abstinence, the THC and synthetic studies suggest that age does not moderate the effects of THC on memory. Additionally, for spatial and non-spatial learning, THC, relative to control, does not affect spatial learning or non-spatial learning in adolescent and adults after prolonged abstinence. It is unclear if age changes this relationship, as interaction effects were inconclusive. There appeared to be a different pattern for neural and behavioral outcomes more closely linked with emotional processes (i.e., fear conditioning and appetitive and aversive information processing), with adolescents showing increased neural and behavioral reductions if exposed to synthetic cannabis. It is unclear if this extends to natural THC, after prolonged abstinence, as this was not explored.

\section{Age, repeated cannabinoid exposure, and cognition during intoxication}

Seven experiments explored the effect of 5-day THC exposure without a prolonged abstinence (minimal or no 
wash-out) period on learning and/or memory. Behavioral testing occurred $30 \mathrm{~min}$ after each injection, except for Moore et al. [55] who exposed rats to THC for 5 days, and then, administered behavioral testing on days 6 and day 10 , 30 minutes after each THC injection.

For spatial and non-spatial learning (Morris Water Maze), Cha et al. [46] administered $2.5 \mathrm{mg} / \mathrm{kg}$ THC, $10.0 \mathrm{mg} / \mathrm{kg}$ THC, or control vehicle to male Sprague Dawley rats in one experiment (experiment 1) and then administered $5.0 \mathrm{mg} /$ $\mathrm{kg}$ in another experiment (experiment 2) with a different sample of male Sprague Dawley rats [46]. In experiment 1, adolescents showed a greater reduction in spatial and nonspatial learning than adults at both dosages, despite no significant differences between adult and adolescent rats who received vehicle injection. For experiment 2 , results for the interaction were inconclusive as statistical results were not presented. Cha et al. [47] extended their design in a mixedgender sample of Sprague Dawley rats across two experiments; in experiment 1 , they administered only one dosage of THC and in experiment 3, they administered three dosages of THC [47]. In experiment 1 , there was a significant moderation of cannabis treatment by age with adolescent rats showing reduced spatial learning compared to adults. However, there was no age by dosage $(2.5 \mathrm{mg} / \mathrm{kg}, 5.0 \mathrm{mg} /$ $\mathrm{kg}$, and $10 \mathrm{mg} / \mathrm{kg}$ ) interaction on spatial learning in the third experiment, suggesting that effects of age on spatial learning are insensitive to THC potency.

In male Sprague Dawley rats, Moore et al. [55] observed that adults pre-treated with THC (5-day THC exposure) showed decreased reductions in spatial learning (Morris Water Maze) on day 6 and 10 after acute intoxication than treatment-matched adolescents. It remains inconclusive if the effects extend to neural mechanisms (e.g., CB1 hippocampal distribution) underlying this behavioral deficit. The interaction was a proposed analysis in their statistical section, but the result was not presented [55]. Nonetheless, this suggests that the age effects of 5-day THC exposure on learning remain evident up to 10 days.

Schramm-Sapyta et al. [56] were interested in whether there are age-related differences in another type of learning-aversive responses to low and high potency of THC. Notably, THC was not paired with an aversive stimulus, but rather was investigated alone as it is thought to have aversive effects at high dosages $(5 \mathrm{mg} / \mathrm{kg})$. The researchers administered $0.5 \mathrm{mg} / \mathrm{kg}, 5 \mathrm{mg} / \mathrm{kg}$, or control vehicle for 5 days to male Sprague Dawley rats. There was a moderating effect of age on conditioned place aversion (cannabis treatment or vehicle control treatment paired with specific locations). Specifically, adolescent and adults both showed greater place aversion after $5 \mathrm{mg} / \mathrm{kg}$ THC administration, relative to control rats, but THC-treated adults spent less time in the drugassociated place than THC-treated adolescents, indicating greater place aversion in adults. There were no age-related differences for the lower dosage. Both age groups showed an increase in taste aversion when treated with THC at both dosages following saccharin, relative to vehicle, but there was a null effect for age by treatment on taste aversion [56]. Additionally, in a separate study of B6 and D2 male mice, there was no main effect of THC treatment on object recognition, measured by the Novel Object Recognition task, across both age groups, and there was a null effect for age by treatment on object recognition [48].

Overall, adolescents appear to be more vulnerable to learning impairment but not memory decline following THC exposure with minimal to no abstinence period. Additionally, it may be that adolescents experience reduced aversive responses to THC, thereby reducing the conditioning effects to neutral cues (i.e., place; as shown in Schramm-Sapyta et al. [56] work). This would indicate that repeated exposure to THC without an abstinence period decreases general learning and increases drug-specific learning for cannabis' positive effects in adolescents at high dosages, compared to adults.

Six studies explored the effect of synthetic cannabinoid exposure without prolonged abstinence, two of which focused on behavior and four of which focused on neuronal changes. Acheson et al. [57] investigated the effect of WIN exposure on spatial memory in adolescent and adult male Sprague Dawley rats. WIN did not affect spatial memory and there was no age by cannabis interaction effect. Fox et al. [58] investigated novelty seeking in male Sprague-Dawley rats $30 \mathrm{~min}$ after WIN exposure. There was no main effect of treatment or an interaction of treatment by age on noveltyseeking behavior, regardless of exposure length ( 1 or 7 days; [58]). The results of these two studies suggest that there is no behaviorally measurable impact of acute WIN intoxication on spatial memory and novelty-seeking regardless of age.

To connect behavioral differences to brain changes, Carvalho et al. [59] exposed male Sprague Dawley rats to WIN for 14 days with a 24-h abstinence period and then measured conditioned place aversion (a behavioral measurement of aversive learning) and neuronal morphology (the shape and structure of neuronal components). Changes in the shape of dendrites were evaluated in the nucleus accumbens and the prefrontal cortex. Only cannabinoid exposed adult rat brains demonstrated increased dendritic length in the medial prefrontal cortex. However, age did not significantly moderate the treatment effect, indicating changes at the neuronal level did not differ between the adult and the adolescent brain. No conclusions could be drawn for any discrepancies in aversion, as authors did not present the results of the interaction analysis [59].

Three studies investigated the effects of synthetic cannabinoids on neuronal activity in the brains of rats after acute intoxication with mixed results. Klugman et al. [60] measured changes in the level of NMDA receptors (subunits NR1 
and NR2b) and Homer protein levels in male Wistar rats. Both are involved in synaptic plasticity and are important for processes such as learning and memory at the cellular level. In both the striatum and medial prefrontal cortex of WIN treated rats, they found a differential effect of age on treatment with an increase in the NR1 subunit in adolescents but a decrease in adults. They also observed a slight reduction in levels of the NR2 subunit in the medial prefrontal cortex, but they did not report the result of the age by treatment interaction. Homer protein levels were also significantly more elevated in adolescents compared to adults [60]. Verdurand et al. [61] investigated the effect of synthetic cannabinoid HU210 on $\mathrm{GABA}_{\mathrm{A}}$ receptor density- the chief inhibitory compound of the brain-after exposure for 1,4 , or 14 days. They observed higher density of $\mathrm{GABA}_{\mathrm{A}}$ receptors in the brains of 4-day treated rats than the 14-day treated rats. However, there was no differential impact of age group [61]. Kang-Park et al. [62] applied WIN to brain slices of Sprague Dawley rats and measured inhibitory and excitatory activity of neurons in the hippocampal CA1 region. Although acute intoxication with WIN significantly decreased the excitatory activity in both adolescent and adult rats, there was not a differential effect on adults and adolescents. However, WINtreated adolescents experienced a greater reduction in inhibitory activity than adults. Reduced inhibition of inhibitory neurotransmission can be understood as increased sensitivity to exogenous cannabinoid-mediated effects at the neuronal level [62]. Overall, all three studies found some evidence for a differential impact of synthetic cannabinoids on neuronal activity based on age.

\section{Discussion}

Our systematic review of human and animal studies specifically aimed to address whether age moderates the relationship between cannabis use and cognition. This question is of particular importance given the scientific debate around whether adolescents, compared to adults, are at heightened risk or are resilient to potential harms of cannabis use and dependence. Integrating both human and animal work, we found preliminary evidence for an age-dependent effect of cannabis that varied based on cannabis use history and intoxication state. However, given the paucity of studies, multiple research gaps exist that need to be addressed in future studies before any strong conclusions can be made.

Interestingly, the human and rodent studies investigating the direct effects of cannabinoid intoxication on cognition showed preliminary evidence for both risk and resilience during adolescence. Human adolescents exhibited less impairment in memory post-intoxication than adults. On the other hand, they also showed greater impaired inhibition and interestingly, less of a craving reduction after intoxication than adults [39]. Similarly, rodent adolescents exhibited increased drug-specific learning for the positive effects of cannabis compared to adults [56]. Combined with reductions in inhibitory control, adolescents' higher craving after cannabis intoxication may promote binge-like behavior, rending adolescents more susceptible to using larger quantities of cannabis over a short period of time than adults. This is in line with research indicating increased prevalence of binge drinking amongst adolescents compared to adults [63] and may be partly driven by adolescents' increased responding to the appetitive vs. aversive effects of drugs [4]. Nevertheless, these findings are extremely tentative given that this is based on one human study. Multiple replication studies are needed before any firm hypotheses can be drawn regarding age-related differences in craving post-cannabis use.

Regarding effects of long-term cannabis use, we found limited evidence that repeated cannabis use is associated with larger impairments in executive functioning and IQ in adolescents compared to adults. It is important to note that this effect may only emerge when comparing almost daily using adolescents and adults. These results align with previous reviews which suggested that early vs. late-onset cannabis use led to increased neurodevelopmental and cognitive disruptions [17, 18, 22]. However, they partially contradict a recent meta-analysis by Scott et al. [19], which found small but comparable decreases in neuropsychological domains in both adolescent and young adult cannabis users overall. Scott et al. [19] compared effect sizes of separate adult and adolescent studies, while we focused solely on studies that made direct comparisons between adolescent and adult cannabis users. This approach is necessary to draw conclusions about whether adolescents are at heightened risk or resilience to the potential effects of cannabis on cognition compared to adults.

Nevertheless, given the weak to moderate level of human evidence, it is too early to draw strong conclusions, especially when the findings of the reviewed animal studies are considered. In line with our human results, adolescent rodents exhibited greater deficits in learning after repeated THC exposure [46, 47, 56]; however, this effect did not emerge in studies that incorporated a prolonged abstinence period [46-50]. This suggests that the effects may be the result of (sub)acute effects of cannabis intoxication and thus, is in line with Scott et al. [19] findings that no impairments emerged in neuropsychological functioning in both adolescents and young adults when studies included a prolonged abstinence period. Moreover, it remains to be tested if the findings reflect direct effects of cannabis on functioning or whether secondary environmental effects play a role. For instance, heavy cannabis use or CUDs may disrupt the ability to pay attention in school, thereby reducing receptiveness to education. This may reflect a malleable reduction in cognition more than a long-lasting neural effect. To unravel the 
underlying mechanisms, extended longitudinal and genetically informed studies targeting various types of cannabis exposure (daily/almost daily; weekly; monthly; past user; never used) as well as age group comparisons (adolescents, adults) are needed.

The current review also included animal studies that investigated the effect of synthetic cannabinoids. Studies investigating behavioral effects found no differential effect of synthetic cannabinoids between adolescents and adults $[57,58]$. However, studies investigating neural effects found differences between adolescents and adult rodents in protein expression and neurotransmitter activity after acute intoxication $[61,63]$. These age-related differences should be interpreted cautiously as it is not possible to infer whether they are evidence of impairment or improvement and whether these findings generalize to other cannabinoids and translate to humans. Synthetic cannabinoids include a broad class of $\mathrm{CB} 1$ and $\mathrm{CB} 2$ receptor agonists and can be more potent than natural cannabinoids. A recent review suggested that its use is increasing, as is the severity of the reported adverse reactions (e.g., hospital visits and symptoms of paranoia) [64]. Unfortunately, human research into the effects of synthetic cannabinoids on cognition is still missing.

In addition to the study of the effects of synthetic cannabinoids in humans, this review highlights multiple research gaps that need to be addressed. First, none of the studies explored the effect of varying ratios of THC to cannabidiol. This is a salient gap as age-related differences in the relationship between cannabis and cognition may vary based on these ratios. Indeed, animal work suggests that long-term cognitive deficits due to cannabis were reversed with treatment of cannabidiol [65]. In addition, human work has suggested that higher cannabidiol strains decreased cannabis-related craving post-intoxication [66]. Therefore, it is possible that adolescents are only more vulnerable to increased craving and drug-related learning at higher potencies of THC $[39,56]$. Similarly, most cannabis research in humans focuses solely on the effects of smoking cannabis; however, oral ingestion of cannabinoids through the use of edibles (e.g., baked goods, candies, drinks) is highly prevalent among both adolescent and adult cannabis users [67, 68]. Importantly, edible cannabis products often have higher concentrations of THC and the metabolic process in the digestive tract leads to higher levels of pharmacologically active THC metabolites in the body than when smoked [69]. Research is needed to elucidate whether agerelated effects of cannabis on cognition differ depending on the route of administration.

Furthermore, research on the effects of cannabis in adolescence should differentiate between developmental periods within adolescence. By treating adolescence unitarily, current research may be missing time windows when risk or resilience patterns shift within the adolescence period. For instance, evidence from alcohol research suggests that the effects of age on social behavior may be most pronounced early adolescent-exposed rats, compared to late adolescentexposed rats [42]. Future rodent and human work would benefit from exploring age-related effects on the relationship between cannabis and cognition through non-linear data modeling strategies. This would allow for insight into whether risk and resilience patterns vary between early, mid, and late adolescence.

Given the methodological constraints and ethical considerations of human studies, especially for adolescents, the value of rodent studies is evident. However, although rodent studies allow us to assess dose-response effects of different cannabinoids and the effect of abstinence on brain and cognition in a way that is not possible in humans, cannabis use-related addictive behaviors remain difficult to study. That is, previous animal studies suggest that rodents do not acquire self-administration behavior with THC [70], despite some evidence suggesting that they do find THC rewarding in other paradigms (e.g., conditioned place preference) [71]. Successful self-administration of synthetic cannabinoid WIN 55,212-2 in Long Evans rats [72] and THC in squirrel monkeys [73] do provide possibilities for future research. However, the challenge of self-administration of THC in rodents further highlights the difficulties of translating findings from animal models to humans given the potential differences in the rewarding and aversive capacity of cannabinoids across species.

While the current review addressed novel questions in both humans and rodents, there are several notable limitations. First, given that some of our studies have 3-way interactions, our null findings may have been due to low power. Secondly, we only had a small sample of human studies $(n=6)$. Therefore, our conclusions should be interpreted cautiously, particularly for human work. Lastly, we only included published studies. Due to publication bias, significant age-related results may be over-represented in this review. Despite these limitations, we believe that our review used a novel approach and question to address whether public health risk for cannabis in terms of cognition is different for adolescents and adults. This review, along with future work, can help inform prevention work such as whether resources should be allocated toward delaying cannabis use if adolescents are indeed at greater risk.

\section{Concluding remarks}

While this systematic review does not offer a conclusive answer to the question of whether age changes the relationship between cannabis and cognition, the novel review question, along with the inclusion of both human and rodent work, has allowed for the formation of important hypotheses 
to be addressed in future work. First, in humans, general executive functioning seems to be more impaired in adolescent, frequent cannabis users compared to adult, frequent cannabis users. Second, in humans, age-effects may be most prominent among very heavy and dependent users, which may suggest CUD-specific effects. Third, in humans, craving and inhibitory control may not decrease as much after cannabis intoxication in adolescents compared to adults. Lastly, in rodents, the age-effects of cannabis on learning appear to be reversible if followed by sustained abstinence. If these hypotheses prove correct, it could lead to important developments in targeted prevention strategies.

Acknowledgements The authors would like to thank Janneke Staaks, MSc, information specialist at the University of Amsterdam for her assistance in developing the search strategy for this review. Additionally, we would like to thank the Fulbright program, supported by the U.S. Department of State's Bureau of Educational and Cultural Affairs and by the Netherlands America Foundation, for fostering the international collaboration that allowed us to work on this paper. This review was supported by grant 1R01 DA042490-01A1 awarded to Janna Cousijn from the National Institute on Drug Abuse/National Institutes of Health.

\section{Compliance with ethical standards}

Conflict of interest All authors have no conflict of interest.

OpenAccess This article is distributed under the terms of the Creative Commons Attribution 4.0 International License (http://creativeco mmons.org/licenses/by/4.0/), which permits unrestricted use, distribution, and reproduction in any medium, provided you give appropriate credit to the original author(s) and the source, provide a link to the Creative Commons license, and indicate if changes were made.

\section{References}

1. Bogt TFM, Looze M, Molcho M et al (2014) Do societal wealth, family affluence and gender account for trends in adolescent cannabis use? A 30 country cross national study. Addiction 2:273-283. https://doi.org/10.1111/add.12373

2. Lubman DI, Cheetham A, Yücel M (2015) Cannabis and adolescent brain development. Pharmacol Ther 148:1-16. https:// doi.org/10.1016/j.pharmthera.2014.11.009

3. Spear LP (2000) The adolescent brain and age-related behavioral manifestations. Neurosci Biobehav Rev 24:417-463. https ://doi.org/10.1016/S0149-7634(00)00014-2

4. Spear LP (2011) Rewards, aversions and affect in adolescence: emerging convergences across laboratory animal and human data. Dev Cogn Neurosci 1:390-403. https://doi.org/10.1016/j. den.2011.08.001

5. Crone EA, Dahl RE (2012) Understanding adolescence as a period of social-affective engagement and goal flexibility. Nat Rev Neurosci 13:636-650. https://doi.org/10.1038/nrn3313

6. Steinberg L (2010) A dual systems model of adolescent risktaking. Dev Psychobiol 52:216-224. https://doi.org/10.1002/ dev. 20445
7. Cousijn J, Luijten M, Feldstein Ewing SW (2018) Adolescent resilience to addiction: a social plasticity hypothesis. Lancet Child Adolesc Health 2:69-78. https://doi.org/10.1016/S2352 -4642(17)30148-7

8. Scallet AC (1991) Neurotoxicology of cannabis and THC: a review of chronic exposure studies in animals. Pharmacol Biochem Behav 40:671-676. https://doi.org/10.1016/00913057(91)90380-K

9. Lynskey MT, Heath AC, Bucholz KK et al (2003) Escalation of drug use in early-onset cannabis users vs co-twin controls. JAMA 289:427-433. https://doi.org/10.1001/jama.289.4.427

10. Pope HGJ, Gruber AJ, Hudson JI et al (2003) Early-onset cannabis use and cognitive deficits: what is the nature of the association? Drug Alcohol Depend 69:303-310

11. Gruber SA, Sagar KA, Dahlgren MK et al (2012) Age of onset of marijuana use and executive function. Psychol Addict Behav J Soc Psychol Addict Behav 26:496-506. https://doi. org/10.1037/a0026269

12. Sariaslan A, Sharp DJ, D'Onofrio BM et al (2016) Long-term outcomes associated with traumatic brain injury in childhood and adolescence: a nationwide swedish cohort study of a wide range of medical and social outcomes. PLoS Med 13:e1002103. https://doi.org/10.1371/journal.pmed.1002103

13. Bryant AL, Schulenberg JE, O'malley PM et al (2003) How academic achievement, attitudes, and behaviors relate to the course of substance use during adolescence: a 6-year, multiwave national longitudinal study. J Res Adolesc 13:361-397

14. Farmer ME, Kittner SJ, Rae DS et al (1995) Education and change in cognitive function. The Epidemiologic Catchment Area Study. Ann Epidemiol 5:1-7

15. Jacobus J, Tapert F S (2014) Effects of cannabis on the adolescent brain. https://www.ingentaconnect.com/content/ben/ cpd/2014/00000020/00000013/art00009. Accessed 10 Aug 2018

16. Batalla A, Bhattacharyya S, Yücel M et al (2013) Structural and functional imaging studies in chronic cannabis users: a systematic review of adolescent and adult findings. PLos One 8:e55821. https://doi.org/10.1371/journal.pone.0055821

17. Lisdahl KM, Gilbart ER, Wright NE, Shollenbarger S (2013) Dare to delay? The impacts of adolescent alcohol and marijuana use onset on cognition, brain structure, and function. Front Psychiatry 4:53

18. Lisdahl KM, Wright NE, Kirchner-Medina C et al (2014) Considering cannabis: the effects of regular cannabis use on neurocognition in adolescents and young adults. Curr Addict Rep $1: 144-156$

19. Scott JC, Slomiak ST, Jones JD et al (2018) Association of cannabis with cognitive functioning in adolescents and young adults: a systematic review and meta-analysis. JAMA Psychiatry 75:585595. https://doi.org/10.1001/jamapsychiatry.2018.0335

20. Crane NA, Schuster RM, Fusar-Poli P, Gonzalez R (2013) Effects of cannabis on neurocognitive functioning: recent advances, neurodevelopmental influences, and sex differences. Neuropsychol Rev 23:117-137. https://doi.org/10.1007/s11065-012-9222-1

21. Blest-Hopley G, Giampietro V, Bhattacharyya S (2018) Residual effects of cannabis use in adolescent and adult brains-a metaanalysis of fMRI studies. Neurosci Biobehav Rev 26-41

22. Ganzer F, Broning S, Kraft S et al (2016) Weighing the evidence: a systematic review on long-term neurocognitive effects of cannabis use in abstinent adolescents and adults. Neuropsychol Rev 26:186-222

23. Moher D, Liberati A, Tetzlaff J et al (2009) Preferred reporting items for systematic reviews and meta-analyses: the PRISMA Statement. PLOS Med 6:e1000097. https://doi.org/10.1371/journ al.pmed.1000097 
24. Livingston MD, Xu X, Komro KA (2016) Predictors of recall error in self-report of age at alcohol use onset. J Stud Alcohol Drugs 77:811-818. https://doi.org/10.15288/jsad.2016.77.811

25. Labouvie E, Bates ME, Pandina RJ (1997) Age of first use: its reliability and predictive utility. J Stud Alcohol 58:638-643. https ://doi.org/10.15288/jsa.1997.58.638

26. de Wit H (2009) Impulsivity as a determinant and consequence of drug use: a review of underlying processes. Addict Biol 14:22-31. https://doi.org/10.1111/j.1369-1600.2008.00129.x

27. Robinson TE, Berridge KC (1993) The neural basis of drug craving: an incentive-sensitization theory of addiction. Brain Res Rev 18:247-291. https://doi.org/10.1016/0165-0173(93)90013-P

28. Rodriguiz RM, Wetsel WC (2006) Assessments of cognitive deficits in mutant mice. In: Levin ED, Buccafusco JJ (eds) Animal models of cognitive impairment. CRC Press, Boca Raton

29. Meier MH, Caspi A, Ambler A et al (2012) Persistent cannabis users show neuropsychological decline from childhood to midlife. PNAS Proc Natl Acad Sci USA 109:E2657-E2664

30. Scott JC, Wolf DH, Calkins ME et al (2017) Cognitive functioning of adolescent and young adult cannabis users in the Philadelphia Neurodevelopmental Cohort. Psychol Addict Behav 31:423-434

31. Lee DC, Stanger C, Budney AJ (2015) A comparison of delay discounting in adolescents and adults in treatment for cannabis use disorders. Exp Clin Psychopharmacol Exp Clin Psychopharmacol 23(130):130-137. https://doi.org/10.1037/a0038792

32. Albertella L, Le Pelley ME, Copeland J (2015) Cannabis use, schizotypy, and negative priming. Psychiatry Res 228:404-410. https://doi.org/10.1016/j.psychres.2015.05.074

33. Rogeberg O (2013) Correlations between cannabis use and IQ change in the Dunedin cohort are consistent with confounding from socioeconomic status. Proc Natl Acad Sci 110:4251-4254. https://doi.org/10.1073/pnas.1215678110

34. Daly M (2013) Personality may explain the association between cannabis use and neuropsychological impairment. Proc Natl Acad Sci 110:E979-E979. https://doi.org/10.1073/pnas.1218571110

35. Moffitt TE, Meier MH, Caspi A, Poulton R (2013) Reply to Rogeberg and Daly: no evidence that socioeconomic status or personality differences confound the association between cannabis use and IQ decline. Proc Natl Acad Sci 110:E980-E982. https://doi. org/10.1073/pnas.1300618110

36. Wampold BE (2015) How important are the common factors in psychotherapy? An update. World Psychiatry 14:270-277. https ://doi.org/10.1002/wps.20238

37. Horvath AO, Del Re AC, Flückiger C, Symonds D (2011) Alliance in individual psychotherapy. Psychotherapy 48:9-16. https ://doi.org/10.1037/a0022186

38. Whelan R, McHugh LA (2009) Temporal discounting of hypothetical monetary rewards by adolescents, adults, and older adults. Psychol Rec 59:247-258. https://doi.org/10.1007/BF03395661

39. Mokrysz C, Freeman TP, Korkki S et al (2016) Are adolescents more vulnerable to the harmful effects of cannabis than adults? A placebo-controlled study in human males. Transl Psychiatry 6:e961. https://doi.org/10.1038/tp.2016.225

40. Treloar HP, Miranda RJ (2018) Using ecological momentary assessment to identify mechanisms of change: an application from a pharmacotherapy trial with adolescent cannabis users. J Stud Alcohol Drugs 79:190-198

41. McCutcheon JE, Marinelli M (2009) Age matters. Eur J Neurosci 29:997-1014. https://doi.org/10.1111/j.1460-9568.2009.06648.x

42. Spear LP (2015) Adolescent alcohol exposure: are there separable vulnerable periods within adolescence? Physiol Behav 122-130

43. Sengupta $P$ (2013) The laboratory rat: relating its age with human's. Int J Prev Med 4:624-630

44. Vardakou I, Pistos C, Spiliopoulou C (2010) Spice drugs as a new trend: mode of action, identification and legislation. Toxicol Lett 197:157-162. https://doi.org/10.1016/j.toxlet.2010.06.002
45. Winstock AR, Barratt MJ (2013) The 12-month prevalence and nature of adverse experiences resulting in emergency medical presentations associated with the use of synthetic cannabinoid products. Hum Psychopharmacol 28:390-393. https://doi. org/10.1002/hup.2292

46. Cha YM, White AM, Kuhn CM et al (2006) Differential effects of delta9-THC on learning in adolescent and adult rats. Pharmacol Biochem Behav 83:448-455

47. Cha YM, Jones KH, Kuhn CM et al (2007) Sex differences in the effects of DELTA9-tetrahydrocannabinol on spatial learning in adolescent and adult rats. Behav Pharmacol 18:563-569

48. Kasten CR, Zhang Y, Boehm SLI (2017) Acute and long-term effects of DELTA9-tetrahydrocannabinol on object recognition and anxiety-like activity are age- and strain-dependent in mice. Pharmacol Biochem Behav 163:9-19

49. O'Shea M, Singh ME, McGregor IS, Mallet PE (2004) Chronic cannabinoid exposure produces lasting memory impairment and increased anxiety in adolescent but not adult rats. J Psychopharmacol (Oxf) 18:502-508

50. O'Shea M, McGregor IS, Mallet PE (2006) Repeated cannabinoid exposure during perinatal, adolescent or early adult ages produces similar long-lasting deficits in object recognition and reduced social interaction in rats. J Psychopharmacol (Oxf) 20:611-621

51. Gleason KA, Birnbaum SG, Shukla A, Ghose S (2012) Susceptibility of the adolescent brain to cannabinoids: long-term hippocampal effects and relevance to schizophrenia. Transl Psychiatry Psychiatry 2:e199

52. Nakamura K (2013) The role of the dorsal raphé nucleus in reward-seeking behavior. Front Integr Neurosci 7:. https://doi. org/10.3389/fnint.2013.00060

53. Samuels ER, Szabadi E (2008) Functional neuroanatomy of the noradrenergic locus coeruleus: its roles in the regulation of arousal and autonomic function part I: principles of functional organisation. Curr Neuropharmacol 6:235-253. https://doi. org/10.2174/157015908785777229

54. Bambico FR, Nguyen N-T, Katz N, Gobbi G (2010) Chronic exposure to cannabinoids during adolescence but not during adulthood impairs emotional behaviour and monoaminergic neurotransmission. Neurobiol Dis 37:641-655

55. Moore NLT, Greenleaf ALR, Acheson SK et al (2010) Role of cannabinoid receptor type 1 desensitization in greater tetrahydrocannabinol impairment of memory in adolescent rats. J Pharmacol Exp Ther 335:294-301

56. Schramm-Sapyta NL, Cha YM, Chaudhry S et al (2007) Differential anxiogenic, aversive, and locomotor effects of THC in adolescent and adult rats. Psychopharmacology 191:867-877

57. Acheson SK, Moore NLT, Kuhn CM et al (2011) The synthetic cannabinoid WIN 55212-2 differentially modulates thigmotaxis but not spatial learning in adolescent and adult animals. Neurosci Lett 487:411-414

58. Fox KM, Sterling RC, Van Bockstaele EJ (2009) Cannabinoids and novelty investigation: influence of age and duration of exposure. Behav Brain Res 196:248-253

59. Carvalho AF, Reyes BAS, Ramalhosa F et al (2016) Repeated administration of a synthetic cannabinoid receptor agonist differentially affects cortical and accumbal neuronal morphology in adolescent and adult rats. Brain Struct Funct 221:407-419

60. Klugmann M, Klippenstein V, Leweke FM et al (2011) "Cannabinoid exposure in pubertal rats increases spontaneous ethanol consumption and NMDA receptor associated protein levels": erratum. J Neuropsychopharmacol 14:519

61. Verdurand M, Dalton VS, Zavitsanou K (2010) GABAA receptor density is altered by cannabinoid treatment in the hippocampus of adult but not adolescent rats. Brain Res 1351:238-245

62. Kang-Park M-H, Wilson WA, Kuhn CM et al (2007) Differential sensitivity of GABAA receptor-mediated IPSCs to cannabinoids 
in hippocampal slices from adolescent and adult rats. J Neurophysiol 98:1223-1230

63. Klugmann M, Klippenstein V, Leweke FM et al (2011) Cannabinoid exposure in pubertal rats increases spontaneous ethanol consumption and NMDA receptor associated protein levels. J Neuropsychopharmacol 14:505-517. https://doi.org/10.1017/S1461 145710001562

64. Loeffler G, Delaney E, Hann M (2016) International trends in spice use: prevalence, motivation for use, relationship to other substances, and perception of use and safety for synthetic cannabinoids. Brain Res Bull 126:8-28. https://doi.org/10.1016/j. brainresbull.2016.04.013

65. Trigo JM, Soliman A, Staios G et al (2016) Sativex associated with behavioral-relapse prevention strategy as treatment for cannabis dependence: a case series. J Addict Med 10:274-279. https ://doi.org/10.1097/ADM.0000000000000229

66. Morgan CJ, Freeman TP, Schafer GL, Curran HV (2010) Cannabidiol attenuates the appetitive effects of 89 -tetrahydrocannabinol in humans smoking their chosen cannabis. Neuropsychopharmacology 35:1879-1885. https://doi.org/10.1038/npp.2010.58

67. Steigerwald S, Wong PO, Cohen BE et al (2018) Smoking, vaping, and use of edibles and other forms of marijuana among U.S. adults. Ann Intern Med 169:890-892. https://doi.org/10.7326/ M18-1681
68. Friese B, Slater MD, Battle RS (2017) Use of marijuana edibles by adolescents in California. J Prim Prev 38:279-294. https://doi. org/10.1007/s10935-017-0474-7

69. Barrus DG, Capogrossi KL, Cates SC et al (2016) Tasty THC: promises and challenges of cannabis edibles. Methods Rep RTI Press 2016

70. Justinova Z, Goldberg SR, Heishman SJ, Tanda G (2005) Selfadministration of cannabinoids by experimental animals and human marijuana smokers. Pharmacol Biochem Behav 81:285299. https://doi.org/10.1016/j.pbb.2005.01.026

71. Ghozland S, Matthes HWD, Simonin F et al (2002) Motivational effects of cannabinoids are mediated by $\mu$-opioid and $\kappa$-opioid receptors. J Neurosci 22:1146-1154. https://doi.org/10.1523/ JNEUROSCI.22-03-01146.2002

72. Fattore L, Cossu G, Martellotta CM, Fratta W (2001) Intravenous self-administration of the cannabinoid CB1 receptor agonist WIN 55,212-2 in rats. Psychopharmacology 156:410-416. https://doi. org/10.1007/s002130100734

73. Justinova Z, Tanda G, Redhi GH, Goldberg SR (2003) Selfadministration of $\Delta \mathrm{A}^{9}$-tetrahydrocannabinol (THC) by drug naive squirrel monkeys. Psychopharmacology 169:135-140. https://doi. org/10.1007/s00213-003-1484-0 